\title{
Variational Procedure of Deriving Diffusion Equation for Spreading in Porous Media
}

\author{
Kira Logvinova \\ Department of Information Systems and Technologies, \\ National Research University Higher School of Economics, Nizhny Novgorod, Russia \\ Email: klogvinova@hse.ru
}

Received July 29, 2013; revised August 29, 2013; accepted September 5, 2013

Copyright (C) 2013 Kira Logvinova. This is an open access article distributed under the Creative Commons Attribution License, which permits unrestricted use, distribution, and reproduction in any medium, provided the original work is properly cited.

\begin{abstract}
We proposed the mathematical model and concrete example of how to use the notion of functional derivatives in order to arrive at a macroscopic equation for dispersion in disordered media. In the sake of simplicity, we considered the case of random process being a Gaussian process.
\end{abstract}

Keywords: Diffusion Equations; Heterogeneous Media; Variational Method

\section{Introduction}

The problem of deriving the governing equations of spreading matters in porous media, derived under different propositions was considered in [1-3]. Thus in [1], we thoroughly used the fact that centered Gaussian processes are completely determined by the (two points) correlation function. A multipoint correlation appears when a linear fixed-point equation is averaged. That each of these correlations splits into a finite (but increasing with the number of points) number of products of correlation functions was quite important for us. It is possible to use the diagrammatic method with more general processes [3]. Then we have much more terms in the expansions. Even if we replace the coefficients of the fixed-point equation for $\mathrm{u}$ by functions of a centered Gaussian process, the method is not of a simple use. It is possible to obtain similar results via a variational method, which we will explain for the example of problem, already considered in $[1,2]$. Not surprisingly, we then will retrieve the already obtained macroscopic equation. Then, we will use the variational method for a variant of equation including functions of a centered Gaussian process.

\section{Mathematical Model}

\subsection{Statement of the Method}

The idea goes back to so-called variational (functional) derivatives [4].

Let $F[\varphi(t)]$ be a functional over function $\varphi(t)$. Then ratio

$$
\frac{\delta F[\varphi]}{\delta \varphi(t)}=\lim _{\Delta t \rightarrow 0} \frac{\{F[\varphi+\delta \varphi]-F[\varphi]\}}{\int_{\Delta t} \mathrm{~d} \tau \delta \varphi(\tau)}
$$

is named a variational (or functional) derivative. It is obvious that this derivative is also a functional that depends on function $\varphi(\tau)$ and point $t$ (as a parameter). In this way we can define the second functional derivative of $F[\varphi(t)]$ with respect to $\varphi(\tau)$ at point $\tau=\tilde{t}$ :

$$
\frac{\delta}{\delta \varphi(\tilde{t})}\left[\frac{\delta F[\varphi]}{\delta \varphi(t)}\right]=\frac{\delta^{2} F[\varphi]}{\delta \varphi(\tilde{t}) \delta \varphi(t)} .
$$

This is again a functional with respect to $\varphi(\tau)$ that depends on the couple of parameters $(t, \tilde{t})$ and so on.

In the case when functional $F[\varphi(t)]=f(\Phi[\varphi(t)])$ depends on functional $\Phi[\varphi(t)]$ (this is the most interesting case for us) the functional derivative satisfies chain rule:

$$
\frac{\delta}{\delta \varphi(\tilde{t})} f(\Phi[\varphi])=\frac{\partial f(\Phi[\varphi])}{\partial \Phi} \frac{\delta \Phi[\varphi]}{\delta \varphi(t)} .
$$

And with $\Phi[\varphi]=F_{1}[\varphi] \cdot F_{2}[\varphi]$, we have

$$
\frac{\delta}{\delta \varphi(\tilde{t})} F_{1}[\varphi] \cdot F_{2}[\varphi]=F_{1}[\varphi] \frac{\delta F_{2}[\varphi]}{\delta \varphi(t)}+F_{2}[\varphi] \frac{\delta F_{1}[\varphi]}{\delta \varphi(t)} .
$$

Also notice that the functional derivative of functional $\varphi\left(\tau_{0}\right)$ with respect to function $\varphi(t)$ satisfies $\frac{\delta \varphi\left(\tau_{0}\right)}{\delta \varphi(t)}=\delta\left(\tau_{0}-t\right)$ and this is very convenient for dif- 
ferentiation procedure.

For following purposes we take the functional $F[\varphi]=\int_{t_{1}}^{t_{2}} \mathrm{~d} \tau L\left(\tau, \varphi(\tau), \frac{\mathrm{d} \varphi(\tau)}{\mathrm{d} \tau}\right)$. Then we will obtain, according to above formulas:

$$
\begin{aligned}
\frac{\delta F[\varphi]}{\delta \varphi(t)} & =\int_{t_{1}}^{t_{2}} \mathrm{~d} \tau\left[\frac{\partial L}{\partial \varphi}+\frac{\partial L}{\partial \dot{\varphi}} \frac{\mathrm{d}}{\mathrm{d} \tau}\right] \frac{\delta \varphi(\tau)}{\delta \varphi(t)} \\
& =\left(-\frac{\mathrm{d}}{\mathrm{d} t} \frac{\partial}{\partial \dot{\varphi}}+\frac{\partial}{\partial \varphi}\right) L\left(t, \varphi(t), \frac{\mathrm{d} \varphi(t)}{\mathrm{d} t}\right)
\end{aligned}
$$

if $t \in\left(t_{1}, t_{2}\right)$.

Functional Taylor's series for functional $F[\varphi(\tau)+\eta(\tau)]$ over function $\eta(\tau)$ about a point $\eta \approx 0$ looks like [4]:

$$
F[\varphi(\tau)+\eta(\tau)]=\exp \left\{\int \mathrm{d} t \eta(t) \frac{\delta}{\delta \varphi(t)}\right\} F[\varphi(\tau)],
$$

where functional shift operator $\exp \left\{\int \mathrm{d} t \eta(t) \frac{\delta}{\delta \varphi(t)}\right\}$ is understood in the sense of expansion over infinite integration limits.

\subsection{Application of Variational Method}

In [1-3] we used the special summation procedure of diagrams of a certain type and received the diffusion equation with fractional derivatives. In this section, we will exploit another method for to receive the self-contained systems of governing equations for diffusion problems that we have used also in [5]. We again consider the spreading of matter in a porous medium such (1) rules the particles transfer on the small scale and we repeat this equation here once more:

$$
\bar{\varepsilon}\left(\frac{\partial u}{\partial t}-D_{0} \frac{\partial^{2} u}{\partial x^{2}}\right)=D_{0} \frac{\partial}{\partial x} \tilde{\varepsilon} \frac{\partial u}{\partial x}-\frac{\partial}{\partial t} \tilde{\varepsilon} u .
$$

Averaging with respect to realizations $\tilde{\varepsilon}(x)$ of the random porosity yields

$$
\bar{\varepsilon}\left(\frac{\partial\langle u\rangle}{\partial t}-D_{0} \frac{\partial^{2}\langle u\rangle}{\partial x^{2}}\right)=D_{0} \frac{\partial}{\partial x}\left\langle\tilde{\varepsilon} \frac{\partial u}{\partial x}\right\rangle-\frac{\partial}{\partial t}\langle\tilde{\varepsilon} u\rangle .
$$

This equation contains the unknown $\langle u\rangle$, and also $\langle\tilde{\varepsilon} u\rangle$ and $\left\langle\tilde{\varepsilon} \frac{\partial u}{\partial x}\right\rangle$. However, the Furutsu-Novikov formula connects the new unknowns to functional derivatives of $\langle u\rangle$ itself, since [4,6-8] the concentration $u$ is a functional of $\tilde{\varepsilon}(x)$. Indeed, we have:

$$
\begin{aligned}
& \langle\tilde{\varepsilon}(x) u\rangle \\
& =\sum_{k=1}^{\infty} \frac{1}{(k-1) !} \int \psi_{k}\left(x, x_{1}^{\prime}, \cdots, x_{k-1}^{\prime}\right)\left\langle f_{k-1}\left(x, x_{1}^{\prime}, \cdots, x_{k-1}^{\prime}\right)\right\rangle \prod_{i=1}^{k-1} \mathrm{~d} x_{i}^{\prime}
\end{aligned}
$$

Here $f_{1}\left(x, x_{1}\right)=\frac{\delta u}{\delta \tilde{\varepsilon}\left(x_{1}\right)}$,

$f_{2}\left(x, x_{1}, x_{2}\right)=\frac{\delta^{2} u}{\delta \tilde{\varepsilon}\left(x_{1}\right) \delta \tilde{\varepsilon}\left(x_{2}\right)}$, and so on,

$f_{k}\left(x, x_{1}, \cdots, x_{k}\right)=\frac{\delta^{k} u}{\delta \tilde{\varepsilon}\left(x_{1}\right) \cdots \delta \tilde{\varepsilon}\left(x_{k}\right)}$ are functional derivatives of increasing order $k$ or of functional $u$, with respect to $\tilde{\varepsilon}$, at points $x_{1}, x_{2}, \cdots, x_{k}$. The functions $\psi_{k}\left(x, x_{1}, \cdots, x_{k-1}\right)$ above are cumulant of random field $\tilde{\varepsilon}(x)$. A similar expression can also be derived for functional $\frac{\partial u}{\partial x}$ instead of $u$ in the equation above

$$
\left\langle\tilde{\varepsilon}(x) \frac{\partial u}{\partial x}\right\rangle=\sum_{k=1}^{\infty} \frac{1}{(k-1) !} \int \psi_{k}\left(x, x_{1}^{\prime}, \cdots, x_{k-1}^{\prime}\right) \frac{\partial}{\partial x}\left\langle f_{k-1}\left(x, x_{1}^{\prime}, \cdots, x_{k-1}^{\prime}\right)\right\rangle \prod_{i=1}^{k-1} \mathrm{~d} x_{i}^{\prime} .
$$

By substituting all above into (1), we derive:

$$
\begin{aligned}
\bar{\varepsilon}\left(\frac{\partial\langle u\rangle}{\partial t}-D_{0} \frac{\partial^{2}\langle u\rangle}{\partial x^{2}}\right)= & D_{0} \frac{\partial}{\partial x} \sum_{k=1}^{\infty} \int \psi_{k}\left(x, x_{1}^{\prime}, \cdots, x_{k-1}^{\prime}\right) \frac{\partial}{\partial x}\left\langle f_{k-1}\left(x, x_{1}^{\prime}, \cdots, x_{k-1}^{\prime}\right)\right\rangle \prod_{i=1}^{k-1} \mathrm{~d} x_{i}^{\prime} \\
& -\sum_{k=1}^{\infty} \frac{1}{(k-1) !} \int \psi_{k}\left(x, x_{1}^{\prime}, \cdots, x_{k-1}^{\prime}\right) \frac{\partial}{\partial x}\left\langle f_{k-1}\left(x, x_{1}^{\prime}, \cdots, x_{k-1}^{\prime}\right)\right\rangle \prod_{i=1}^{k-1} \mathrm{~d} x_{i}^{\prime}
\end{aligned}
$$

We see here that together with averaged concentration $\langle u\rangle$ we have also averaged values of functional derivatives for $u$ of different orders: $\left\langle f_{k-1}\right\rangle(k=1,2, \cdots)$. By taking the functional derivative of (1) with respect to $\tilde{\varepsilon}$ at point $x_{1}$, we obtain

$$
\begin{aligned}
& \bar{\varepsilon}\left(\frac{\partial f_{1}}{\partial t}-D_{0} \frac{\partial^{2} f_{1}}{\partial x^{2}}\right) \\
& =D_{0} \frac{\partial}{\partial x}\left[\delta\left(x-x_{1}\right) \frac{\partial u}{\partial x}-\tilde{\varepsilon} \frac{\partial f_{1}}{\partial x}\right]-\frac{\partial}{\partial t}\left[\delta\left(x-x_{1}\right) u+\tilde{\varepsilon} f_{1}\right]
\end{aligned}
$$


Here $f_{1}\left(x, x_{1}\right)=\frac{\delta u}{\delta \tilde{\varepsilon}\left(x_{1}\right)}$ in turn is a random functional $\tilde{\varepsilon}(x)$, with two free coordinates which are $x, x_{1}$. By averaging (2) over random realizations of $\tilde{\varepsilon}(x)$, we receive the following equation for $\left\langle f_{1}\left(x, x_{1}\right)\right\rangle$ :

$$
\begin{aligned}
& \bar{\varepsilon}\left(\frac{\partial\left\langle f_{1}\right\rangle}{\partial t}-D_{0} \frac{\partial^{2}\left\langle f_{1}\right\rangle}{\partial x^{2}}\right) \\
= & D_{0} \frac{\partial}{\partial x}\left[\delta\left(x-x_{1}\right) \frac{\partial\langle u\rangle}{\partial x}-\left\langle\tilde{\varepsilon} \frac{\partial f_{1}}{\partial x}\right\rangle\right] \\
& -\frac{\partial}{\partial t}\left[\delta\left(x-x_{1}\right)\langle u\rangle+\left\langle\tilde{\varepsilon} f_{1}\right\rangle\right]
\end{aligned}
$$

This equation contains the unknown function $\left\langle f_{1}\left(x, x_{1}\right)\right\rangle$ and also new unknown functions, which are $\left\langle\tilde{\varepsilon} f_{1}\right\rangle$ and $\left\langle\tilde{\varepsilon} \frac{\partial f_{1}}{\partial x}\right\rangle$. If we apply Furutsu-Novikov formula to functionals $f_{1}$ and $\frac{\partial f_{1}}{\partial x}$ instead of $u$, then substituting the results into (3), we find: (see below (4))

We should have written here "and so on", since after that we should take the functional derivative over $\tilde{\varepsilon}\left(x_{2}\right)$ and then average the obtained equations over the realizations of $\tilde{\varepsilon}$ and apply Furutsu-Novikov's formula again. As a result, we would obtain an equation for $\left\langle f_{2}\left(x, x_{1}, x_{2}\right)\right\rangle$. Iteratively using the procedure would lead us to an infinite system of equations for the sequence of functions $\left\langle f_{k}\right\rangle(k=0,1, \cdots)$, if we set $\left\langle f_{0}\right\rangle=\langle u\rangle$.

The structure of the thus obtained equations is such that the $k$-th equation in the hierarchy, with unknown $\left\langle f_{k}\right\rangle$ on the left hand-side has $\left\langle f_{k+1}\right\rangle,\left\langle f_{k+2}\right\rangle$, and the concentration $\langle u\rangle$ in its right hand-side. And we are led to set the natural question "When can we break this chain of equations?". It turns out that under quite reasonable assumptions, this problem can be solved.

Here we consider random media, where cumulant functions entering Equation (4) are of the even order $\psi_{2 m} \sim a_{0}^{2 m}$ where $a_{0}$ denotes the amplitude of the telegraph or normal random field $\tilde{\varepsilon}$. Here we should assume that in a porous media model, and due to the definition of the porosity $\tilde{\varepsilon}(x)$ we should take for granted that $a_{0}<1$. Considering the fluctuations to be weak, we neglect cumulant of the order higher than two. Moreover, each time $\tilde{\varepsilon}(x)$ is a centered Gaussian process, all the cumulant are equal to zero, except $\psi_{2}$ which also is to the two-point correlation function of process $\tilde{\varepsilon}$. That is why in (4) and as well as in subsequent equations, which are not given here, we can leave only the addends containing the second order correlation functions. In this case, instead of above we get, respectively:

$$
\begin{gathered}
\bar{\varepsilon}\left(\frac{\partial\langle u(x, t)\rangle}{\partial t}-D_{0} \frac{\partial^{2}\langle u(x, t)\rangle}{\partial x^{2}}\right) \frac{1}{2} \\
=D_{0} \frac{\partial}{\partial x} \int \psi_{2}\left(x, x^{\prime}\right) \frac{\partial}{\partial x}\left\langle f_{1}\left(x, x^{\prime}\right)\right\rangle \mathrm{d} x^{\prime} \\
-\int \psi_{2}\left(x, x^{\prime}\right) \frac{\partial}{\partial t}\left\langle f_{1}\left(x, x^{\prime}\right)\right\rangle \mathrm{d} x^{\prime} \\
\bar{\varepsilon}\left(\frac{\partial\left\langle f_{1}\left(x, x_{1}\right)\right\rangle}{\partial t}-D_{0} \frac{\partial^{2}\left\langle f_{1}\left(x, x_{1}\right)\right\rangle}{\partial x^{2}}\right) \\
=D_{0} \frac{\partial}{\partial x}\left[\delta\left(x-x_{1}\right) \frac{\partial\left\langle u\left(x, x_{1}\right)\right\rangle}{\partial x}\right]-\frac{\partial}{\partial t}\left[\delta\left(x-x_{1}\right)\left\langle u\left(x, x_{1}\right)\right\rangle\right] \\
+D_{0} \frac{\partial}{\partial x} \int \psi_{2}\left(x, x^{\prime}\right) \frac{\partial}{\partial x}\left\langle f_{2}\left(x, x_{1} x^{\prime}\right)\right\rangle \mathrm{d} x^{\prime} \\
-\int \psi_{2}\left(x, x^{\prime}\right) \frac{\partial}{\partial t}\left\langle f_{2}\left(x, x_{1}, x^{\prime}\right)\right\rangle \mathrm{d} x^{\prime}
\end{gathered}
$$

We mentioned that if porosity represents itself a normal random field, then (5) is exact because all cumulant except one are zero. As we consider small porosity fluctuations, then in the right-hand side of (5) the third and the fourth addend in the right-hand part, containing $\left\langle f_{2}\left(x, x_{1}, x^{\prime}\right)\right\rangle$, will be neglected because they are proportional to $a_{0}^{4}$. Since also the functional derivative of $\tilde{\varepsilon}(x)$, computed respect to $\tilde{\varepsilon}$ itself at $x^{\prime}$ is $\delta\left(x-x^{\prime}\right)$ we arrive at the following system of equations, which turns out to be closed and has the following form:

$$
\begin{aligned}
& \bar{\varepsilon}\left(\frac{\partial\langle u\rangle}{\partial t}-D_{0} \frac{\partial^{2}\langle u\rangle}{\partial x^{2}}\right) \\
= & D_{0} \frac{\partial}{\partial x} \int \psi_{2}\left(x, x^{\prime}\right) \frac{\partial\left\langle f_{1}\right\rangle}{\partial x} \mathrm{~d} x^{\prime}-\int \psi_{2}\left(x, x^{\prime}\right) \frac{\partial\left\langle f_{1}\right\rangle}{\partial t} \mathrm{~d} x^{\prime}
\end{aligned}
$$




$$
\begin{aligned}
& \bar{\varepsilon}\left(\frac{\partial\left\langle f_{1}\right\rangle}{\partial t}-D_{0} \frac{\partial^{2}\left\langle f_{1}\right\rangle}{\partial x^{2}}\right) \\
= & D_{0} \frac{\partial}{\partial x}\left[\delta\left(x-x^{\prime}\right) \frac{\partial\langle u\rangle}{\partial x}\right]-\delta\left(x-x^{\prime}\right) \frac{\partial\langle u\rangle}{\partial t}
\end{aligned}
$$

where $\langle u(x, t)\rangle$ is the averaged concentration, while $f_{1}\left(x, x^{\prime}, t\right)=\frac{\delta u}{\delta \tilde{\varepsilon}\left(x^{\prime}\right)}$ is the first functional derivative of the concentration, with respect to $\tilde{\varepsilon}\left(x^{\prime}\right)$. And $\psi_{2}\left(x, x^{\prime}\right)$ denotes the correlation function of second order of random field $\tilde{\varepsilon}(x)$. System (6)-(7) will be solved in the unbounded domain $-\infty<x<\infty$, starting from the following initial condition:

$$
\langle u(x, 0)\rangle=u(x, 0)=\varphi(x)
$$

This leads us immediately to the following initial condition for $\left\langle f_{1}\left(x, x^{\prime}, t\right)\right\rangle$ :

$$
\left\langle f_{1}\left(x, x^{\prime}, 0\right)\right\rangle=0 \text {. }
$$

Indeed, we have $f_{1}\left(x, x^{\prime}, 0\right)=\frac{\delta u(x, 0)}{\delta \tilde{\varepsilon}\left(x^{\prime}\right)}=\frac{\delta \varphi}{\delta \tilde{\varepsilon}\left(x^{\prime}\right)}=0$ since the initial concentration $\varphi(x)$ is determined and independent of $\tilde{\varepsilon}\left(x^{\prime}\right)$.

In order to give the integrals on the right hand-side of (6) a concrete meaning, we now specialize the Gaussian process $\tilde{\varepsilon}(x)$ by assigning a definite expression to its correlation function.

\section{Basic Equation Evolution}

Let us assume that the random field $\tilde{\varepsilon}(x)$ is homogeneous and isotropic. Then, without any concrete definition of the correlation function $\Psi_{2}$, let us note that the latter depends only on the modulus of its arguments' difference $\Psi_{2}\left(x x^{\prime}\right)=\Psi_{2}\left(\left|x-x^{\prime}\right|\right)$.

Our ultimate aim is to derive an equation related only to the mean concentration $\langle u\rangle$. The procedure of getting such as equation seems evident. It is clearly seen from (7) that $\left\langle f_{1}\left(x, x^{\prime}, t\right)\right\rangle$ are generated by the concentration that appears in the right-hand part of this equation. The function $\left\langle f_{1}\left(x, x^{\prime}, t\right)\right\rangle$ can be found using the Green's function of operator $\frac{\partial}{\partial t}-D_{0} \frac{\partial}{\partial x^{2}}$. Then, after substitute-

ing the obtained solution for $\left\langle f_{1}\left(x, x^{\prime}, t\right)\right\rangle$ into (6), we get the final integro-differential equation for $\langle u\rangle$.

However, such a procedure is rather intricate in $(x, t)$ representation. In (6) and (7) it is more convenient to turn to $(q, k)$-representation, i.e. to use Laplace transform with respect to time ( $q$-parameter) and Fourier transform over with respect to space ( $k$-parameter). After all the necessary calculations instead of (6) and (7), we get, re- spectively:

$$
\begin{aligned}
& \left(q+D_{0} k_{0}^{2}\right)\left\langle n\left(k_{0}\right)\right\rangle \\
= & \varphi\left(k_{0}\right)-\frac{2 \pi}{\bar{\varepsilon}} \int \Psi(k)\left[q+D_{0} k_{0}\left(k_{0}-k\right)\right]\left\langle f_{1}\left(k_{0}-k, k\right)\right\rangle \mathrm{d} k
\end{aligned}
$$

$$
\begin{aligned}
& \left(q+D_{0} k_{0}^{2}\right)\left\langle f_{1}\left(k_{0}, k\right)\right\rangle \\
= & \frac{\varphi\left(k_{0}+k\right)}{2 \pi \bar{\varepsilon}}-\frac{1}{2 \pi \bar{\varepsilon}}\left[q+D_{0} k_{0}\left(k_{0}+k\right)\right]\left\langle u\left(k_{0}+k\right)\right\rangle
\end{aligned}
$$

In the last relations the argument $q$ of $\left\langle u\left(q, k_{0}\right)\right\rangle$ and $f_{1}\left(q, k_{0}, k\right)$ is omitted for the sake of simplicity. We also have

$$
\Psi_{2}\left(\left|x-x^{\prime}\right|\right)=\int \Psi_{2}(k) \mathrm{e}^{i k\left|x-x^{\prime}\right|} \mathrm{d} k
$$

and

$$
\left\langle f_{1}\left(k_{1}, k_{2}\right)\right\rangle=\frac{1}{(2 \pi)^{2}} \int\left\langle f_{1}\left(x, x^{\prime}\right)\right\rangle \mathrm{e}^{-i k_{1} x-i k_{2} x^{\prime}} \mathrm{d} x \mathrm{~d} x^{\prime} .
$$

Also note, that if we choose $k_{0}-k$ in (11) instead of argument $k_{0}$, then we get the following equation for function $\left\langle f_{1}\left(k_{0}-k, k\right)\right\rangle$ appearing in (10):

$$
\begin{aligned}
& {\left[q+D_{0}\left(k_{0}-k\right)^{2}\right]\left\langle f_{1}\left(k_{0}-k, k\right)\right\rangle } \\
= & \frac{\varphi\left(k_{0}\right)}{2 \pi \bar{\varepsilon}}-\frac{1}{2 \pi \bar{\varepsilon}}\left[q+D_{0} k_{0}\left(k_{0}-k\right)\right]\left\langle u\left(k_{0}\right)\right\rangle .
\end{aligned}
$$

From this we compute $\left\langle f_{1}\left(k_{0}-k, k, q\right)\right\rangle$. Substituting in (10) the thus obtained expression, we get an algebraic equation with respect to $\left\langle u\left(k_{0}, q\right)\right\rangle$ :

$$
\left[q+D_{0} k_{0}^{2}-B\left(k_{0}\right)\right]\left\langle u\left(k_{0}\right)\right\rangle=\varphi\left(k_{0}\right)\left(1-A\left(k_{0}\right)\right)
$$

with $\mathrm{A}$ and $\mathrm{B}$ being defined by

$$
\begin{gathered}
A\left(k_{0}\right)=\frac{1}{\bar{\varepsilon}^{2}} \int \frac{q+D_{0} k_{0}\left(k_{0}-k\right)}{q+D_{0}\left(k_{0}-k\right)^{2}} \Psi_{2}(k) \mathrm{d} k \\
B\left(k_{0}\right)=\frac{1}{\bar{\varepsilon}^{2}} \int \frac{\left[q+D_{0} k_{0}\left(k_{0}-k\right)\right]^{2}}{q+D_{0}\left(k_{0}-k\right)^{2}} \psi_{2}(k) \mathrm{d} k
\end{gathered}
$$

Thus, we have explicit solutions to (6) and (7) in $(q, k)$-representation. Therefore, Equation $(12)$ in $(x, t)$ representation is equivalent to basic equation [1], which we reproduce here:

$$
\begin{aligned}
& \frac{\partial\langle u(x, t)\rangle}{\partial t}-D_{0}(1-\lambda) \frac{\partial^{2}\langle u(x, t)\rangle}{\partial x^{2}} \\
= & \tau^{1 / 2} D_{t}^{1 / 2}\left[D_{0}(3-2 \lambda) \frac{\partial^{2}\langle u(x, t)\rangle}{\partial x^{2}}-(3-\lambda) \frac{\partial\langle u(x, t)\rangle}{\partial t}\right]
\end{aligned}
$$

Hence successive approximation method and functional derivative method lead us to similar results for problems 
such that both methods are available.

\section{Conclusion}

So, in this paper, we presented an example of how to use the notion of a functional derivative in order to arrive at a macroscopic equation for dispersion in disordered media. In fact, we also used the present method for equation, which had been derived for a different type of disordered medium, made of inter-twisted tubes, such that a onedimensional approach has physical meaning. Hence, the example can only serve formally for two reasons. Indeed, the sample paths of Gaussian processes can take negative values, which are not good when the existence of solutions is needed. The drawback can be removed by considering $\varepsilon$ being replaced by the exponential of a Gaussian process. We will not do it, but the presented method works fairly well for this case and gives the results already obtained via Feynman diagrams in our work [1].

\section{REFERENCES}

[1] K. Logvinova and O. Kozyrev, "Models for the Average of the Solutions to a P.D.E. with Stochastic Coefficients," European Journal of Scientific Research, Vol. 45, No. 3, 2010, pp. 383-390.
[2] O. Kozyrev and K. Logvinova, "Small Scale Models for the Spreading of Matter in Disordered Porous Media," European Journal of Scientific Research, Vol. 45, No. 1, 2010, pp. 64-78.

[3] O. Kozyrev, "Diffusion Fractional Models for a Complex Porous Media in a Random Force Field for 3D Case," European Journal of Scientific Research, Vol. 95, No. 3, 2013, pp. 436-443.

[4] V. I. Klyatskin "Waves and Stochastic Equations in Randomly Inhomogeneous Media," Edition de Physique, Paris, 1985.

[5] K. Logvinova and M.-C. Neel, "A Fractional Equation for Anomalous Diffusion in a Randomly Heterogeneous Porous Media," Chaos, Vol. 14, No. 4, 2004, pp. 982-987. http://dx.doi.org/10.1063/1.1796211

[6] E.A. Novikov, "Functionals and Random Force Methods for Turbulence Theory," Journal of Experimental and Theoretical Physics, Vol. 47, No. 5, 1964, p. 1919.

[7] M. Donsker, "On Function Space Integrals," Proceedings of a Conference on the Theory and Applications of Analysis in Function Space, M.II. Press, Cambridge, 1964, pp. 17-30.

[8] K. Furutsu, "On the Statistical Theory of Electromagnetic Waves in a Fluctuating Medium," Journal of Research of the National Bureau of Standards, Vol. 67D, No. 3, 1963, p. 303. 